\title{
What controls rates of evolution?
}

EVER since the pioneer work of G. G. Simpson some three decades ago it has been recognised that one of the most important contributions that palaeontology can make to our knowledge and understanding of evolution is in determining rates of morphological change for different fossil groups over long periods of time. Simpson was the first to bring comparative quantitative data to bear on this subject, and convincingly demonstrated that, in terms of rates of origination and extinction, and of structural innovation, the mammals easily led the field. Van Valen has now endeavoured to show, in a letter published on page 298 of this issue, that whereas in general mammals have evolved at a faster rate than other organisms, there are at least two types of evolution in which the rate is effectively the same, namely the rate of amino acid substitution in proteins and change of size measured linearly. The first type of evolution is termed the epistandard, the other the standand mode.

Van Valen attempts a tentative explanation of the epistandard mode in terms of his Red Queen hypothesis, which is spelled out fully in an earlier paper (Evolution Theory, 1, 1; 1973). Readers of Through the Looking Glass will recall that the Red Queen explained to Alice that it took all the running one can do to stay in the same place. Van Valen put forward his hypothesis in an attempt to explain why the probability of extinction of fossil groups was more or less constant in time. It is based on the idea that all species within a given adaptive zone compete intensively. A successful adaptive response by one species is assumed to accur at the expense of other species, which must either adapt by themselves speciating or become extinct, as the 'quality' of their environment is reduced. This phenomenon leads to an endless chain of adaptive responses and in the long run means that fitness and rate of extinction remain constant.

The high rate of diversification and evolutionary turnover in mammals is likely to be the result of a variety of factors, such as strong competitive interactions leading to specialisation in feeding methods, limitations on food supply, high mobility and energy use, interspecies aggression and territoriality. Such factors will conspire to lower the 'resource threshold' needed to prevent extinction, compared with other animals. Epistandard rates are required to make up the losses through extinction.

Though the Red Queen model might apply well to mammals, there are doubts about its more general validity. This is brought out well, for instance, in a thoughtful paper by Stanley (Systematic Zool., 22, 486; 1973), who analyses the effects of competition on evolutionary rates. Attention is confined mostly to the mammals and the bivalve molluscs (that is, clams and oysters). In sharp contrast to mammals, the bivalves are nearly all sea-bed suspension feeders which mind their own business, characterised by weak interactions with other species, primitive inflexible behaviour, uncrowded, largely sedentary mode of life and generalised feeding habits.

This has as a direct consequence substantially lower rates of evolution than mammals, as Stanley amply documents. Limits on the bivalve populations are imposed more by predation and fluctuations in the physical environment than by food resources, and biological competition is minimal. As Stanley observes laconically, "Interspecific aggression is not characteristic of bivalve behaviour". What is true of bivalves is without much doubt true of the majority of benthonic invertebrates. Through the Looking Glass might be less appropriate as a textual source for the relevant evolutionary sermon than The Walrus and the Carpenter. A. HallaM experiments, the cDNA anneals back to its poly $(\mathrm{A})^{+} \mathrm{mRNA}$ template, but does not react with the poly $(\mathbf{A})^{-}$mRNA until much higher $R_{0} t$ values are reached (and perhaps only then because of the presence of contaminating poly(A) ${ }^{+}$mRNA in the $\operatorname{poly}(\mathrm{A})^{-}$fraction). Because the cDNA represents only the 500 or so bases at the $3^{\prime}$ terminal end of the messenger, immediately adjacent to the poly(A), the hybridisation analysis does not in itself exclude the possibility that the poly $(\mathrm{A})^{-}$mRNA represents $5^{\prime}$ ends broken off poly $(\mathrm{A})^{+}$ messengers; but this is rendered rather unlikely by the observation that $\operatorname{poly}(\mathrm{A})^{+}$and $\operatorname{poly}(\mathrm{A})^{-}$messengers are of the same size.

A similar situation is found in early sea urchin embryos, in which the sequences present in the $\operatorname{poly}(\mathrm{A})^{+}$and poly(A)mRNA fractions were investigated by preparing a cDNA from the poly(A) ${ }^{+}$mRNA by using the DNA polymerase I enzyme of Escherichia coli. At the maximum $R_{0} t$ values reached, some $80 \%$ of this cDNA annealed with the $\operatorname{poly}(\mathrm{A})^{+}$mRNA; the low level of reaction with the poly $(A)^{-}$mRNA shows that this fraction is almost entirely constituted of sequences different from those present in the poly $(\mathrm{A})^{+}$mRNA class. From the hybridisation reaction between the cDNA and poly $(\mathrm{A})^{+}$mRNA, it is possible to calculate that the complexity of the poly $(\mathrm{A})^{+}$mRNA corresponds to about 1,400 different species, each present on average 10,000 times in the embryo (which at this stage corresponds to about 400 cells). The complexity and abundance of the poly $(\mathrm{A})^{-}$class cannot be estimated since no probe for its sequences has been developed. Since the earlier work of Galau et al. (Cell, 2, 9-22; 1974) showed that the total polysomal mRNA falls into two abundance classes at the 600 cell stage, with less than $10 \%$ of the
mRNA representing 14,000 sequences present on average 340 times in the embryo, while most of the mRNA represents a much smaller number of sequences present in many copies each, it is of obvious importance to define the complexity of the poly(A) mRNA and to see how the total messenger population is divided into classes containing and lacking poly(A).

Only the histone messengers previously have been shown to lack poly(A), a feature which it was thought might be related to their synthesis during only part of the cell cycle; and also which it seemed possible might be responsible for their rapid appearance in the cytoplasm after addition of a radioactive label-the delay in appearance of a label in poly(A) ${ }^{+}$mRNA might be due to the time involved in addition of $\operatorname{poly}(\mathrm{A})$. The kinetics of production and decay of the poly(A) ${ }^{+}$and poly $(\mathrm{A})^{-}$mRNA classes in the HeLa cell, however, seem to be indistinguishable, so that whatever mechanism is used to produce the poly $(\mathrm{A})^{--}$class does not promote a more rapid cytoplasmic transport. Nothing is known yet about the mechanism of production of poly $(\mathrm{A})^{-}$messages, whether a large precursor is involved as seems to be the case with poly $(\mathrm{A})^{+}$mRNA or whether some different mechanism is implicated. The existence of poly(A) $)^{-}$mRNA, however, appears to argue against models which suppose that the addition of poly(A) provides the only mechanism by which messengers are recognised for transport to the cytoplasm; and also raises the question of what function the poly(A) may play in the cytoplasmic activities of the messengers containing it. The purpose of poly(A) is therefore now less clear than ever.

BenJamin Lewin 